\title{
Effects on Bacillus subtilis of conditional expression of the accBC operon encoding subunits of acetyl coenzyme $A$ carboxylase, the first enzyme of fatty acid synthesis
}

\author{
Carlos A. Perez, Patricia Marini and Diego de Mendoza \\ Author for correspondence: Diego de Mendoza. Tel: +54 41350661 . Fax: +54 41390465 . \\ e-mail: ddemendo@unromb.edu.ar
}

\section{Programa}

Multidisciplinario de Biología Experimental (PROMUBIE) and Departamento de Microbiologia, Facultad de Ciencias Bioquímicas y Farmacéuticas, Universidad Nacional de Rosario, Suipacha 531, 2000Rosario, Argentina

\begin{abstract}
A Bacillus subtilis strain was constructed in which the operon accBC, encoding the biotin carboxyl carrier protein (BCCP) and biotin carboxylase (BC) subunits of acetyl-COA carboxylase (ACC), was placed under the control of the IPTGinducible promoter spac. A reduction in the levels of BCCP resulted in a decrease of de novo fatty acid synthesis and in the total content of membrane fatty acids. This strain was dependent upon the presence of IPTG for a normal growth phenotype. Growth was specifically restored by supplying exogenous long branched-chain fatty acids in the medium, indicating that the inducerdependent phenotype was specifically related to a conditional block in fatty acid biosynthesis. The strain showed a strong decrease in sporulation frequency when it was induced to sporulate in an IPTG-free medium. Germination and outgrowth were both delayed in spores of the mutant obtained in the absence of IPTG.
\end{abstract}

Keywords: Bacillus subtilis, fatty acid synthesis, acetyl-CoA carboxylase, cell cycle

\section{INTRODUCTION}

Acetyl-CoA carboxylase (ACC, EC 6.4.1.2) plays a pivotal role in the biosynthesis of fatty acids. It catalyses the first committed step of fatty acid synthesis, the synthesis of malonyl-CoA. The overall reaction consists of two distinct half-reactions, the carboxylation of biotin with bicarbonate and the transfer of the $\mathrm{CO}_{2}$ group from carboxy-biotin to acetyl-CoA to form malonyl-CoA. In Escherichia coli, the half-reactions are catalysed by two different ACC subunits, biotin carboxylase (BC) and carboxyl transferase (CT). A third subunit, biotin carboxyl carrier protein (BCCP), carries the essential biotin cofactor covalently bound to a lysine residue proximal to its carboxyl terminus ( $\mathrm{Li} \&$ Cronan, 1992a, b; Magnuson et al., 1993).

We have recently cloned and characterized the Bacillus subtilis genes encoding two subunits of ACC, BCCP and BC (Marini et al., 1995). The B. subtilis accB gene, which encodes BCCP, is part of an operon that includes acc C, the gene encoding the $\mathrm{BC}$ subunit of ACC. We also found that the $17 \cdot 2 \mathrm{kDa}$ BCCP subunit from $B$. subtilis is able to interact with other subunits of the E. coli ACC as

Abbreviations: $A C C$, acetyl-CoA carboxylase; $B C C P$, biotin carboxyl carrier protein; $B C$, biotin carboxylase; $C T$, carboxyl transferase. well as the E. coli biotin apoprotein ligase that biotinylates BCCP (Marini et al., 1995).

Since ACC catalyses the first committed step of fatty acid synthesis, it has been the subject of intensive study (Volpe \& Vagelos, 1976; Wakil et al., 1983). Several mechanisms have been proposed for the regulation of mammalian ACC. These include allosteric regulation by citrate and isocitrate (López-Casilla et al., 1987), feedback inhibition by the end product, long chain acyl-CoA (Nikawa et al., 1979), and modification by phosphorylation and dephosphorylation (Kim, 1983). Recently, regulation of ACC at the level of gene expression has also been demonstrated in mouse preadipocytes (Luo \& Kim, 1990). Unlike its eukaryotic counterpart, almost no information is available on the regulation of ACC in E. coli and other prokaryotes (Magnuson et al., 1993; Rock \& Cronan, 1996). Physiological studies to determine the rate-limiting step in E. coli phospholipid biosynthesis have led to the suggestion that ACC is the controlling step (for a review see Magnuson et al., 1993). Furthermore, recent studies indicate that the $E$. coli ACC is regulated by growth rate (Li \& Cronan, 1993).

In bacteria, fatty acids are primarily precursors of phospholipids rather than storage fuels, and so ACC activity is coordinated with cell growth and division. $B$. 
subtilis, in addition, in response to conditions of nutrient limitation enters a developmental process that culminates in the formation of spores (for a review see Errington, 1993). Spore formation in this organism (and also spore germination and outgrowth) constitutes a differentiation process that has long been appreciated as a model for investigation of prokaryotic development (for reviews see Errington, 1993; Foster \& Johnstone, 1990; Moir \& Smith, 1990). This cellular life cycle involves a variety of biochemical and physiological events, including membrane differentiation accompanied by phospholipid synthesis (Piggot et al., 1994), that must start with the reaction catalysed by ACC. This feature, combined with the fact that ACC has been postulated as a primary site for regulation of lipid synthesis (Magnuson et al., 1993), suggests that its activity could be important during sporulation, providing a link between intracellular lipid levels and the differentiation process. However, the study of the role of ACC in the B. subtilis cell cycle is hampered by the fact that the activity of this enzyme is probably essential for growth and that viable mutants of ACC are not yet available. As a first step in the study of the role(s) ACC plays in the cell, the $B$. subtilis accBC operon was placed under the control of the IPTG-inducible promoter Pspac. This mutant was used to analyse the effect of conditional expression of $a c c B C$ on growth and differentiation.

\section{METHODS}

Bacterial strains and plasmids. B. subtilis was propagated in DS medium (Schaeffer et al., 1965). The parental strain was JH642 (trpC2 pheA1). Strain DL2, carrying the integrated plasmid that introduces the $a c c B C$ operon under the control of the spac promoter, was obtained by transformation as described by Spizizen (1958). This strain was grown in medium containing $5 \mu \mathrm{g}$ chloramphenicol $\mathrm{ml}^{-1}$ (final concentration). Plasmid pDH88, suitable for placing genes under control of the inducible spac promoter in B. subtilis, was described by Henner (1990). Plasmid pHlacl, containing the lacl gene under the control of the Bacillus licheniformis penicillinase transcriptional and translational control signals (Henner, 1990) on the multicopy plasmid pHT1030 (Arantes \& Lereclus, 1991), was the generous gift of $\mathrm{T}$. Msadek (Unité de Biochimie Microbienne, Institut Pasteur, Paris, France).

DNA methods. Plasmid pDL1 (Fig. 1) was constructed as follows. Firstly the oligomers 5'-AATTTCAAGCTTAGGAGTGCGATTGAAATGTTAAATATC- $3^{\prime}$ and $5^{\prime}$-AAGCTTCATGGATCCTACAATGCAGACAACTGTGTTTTC- $3^{\prime}$ were synthesized so that PCR could be used to amplify a chromosomal segment of DNA containing a promoterless 397 bp fragment of accBC (coordinates 320-717 in Marini et al., 1995). This fragment contains 26 nucleotides preceding the accBC initiation codon, which includes the natural ribosomebinding site (RBS), and extends through the first 124 of the 159 codons of the $a c c B$ gene. Oligomers were synthesized so that the 397 bp product contained a HindIII site preceding the $a c c B$ RBS and a BamHI site close to the end of the fragment. The PCR product was cloned into the HindIII-BglII sites of pDH88, giving rise to $\mathrm{pDL} 1$ (Fig. 1).

Total DNA was isolated from B. subtilis strains grown in LB medium (Sambrook et al., 1989) as described by Gardiol et al. (1993). Southern blots were performed applying stringent hybridization and washing conditions (Sambrook et al., 1989).
An internal 300 bp EcoRI-HindIII fragment of $a c c B$ (see Fig. 1 and Marini et al., 1995) labelled with ${ }^{32} \mathrm{P}$ by the random priming method (Sambrook et al., 1989) was used as a DNA probe.

Detection of BCCP. Cells were grown in DS medium and labelled with $\left[{ }^{3} \mathrm{H}\right]$ biotin as previously described (Marini et al., 1995). After harvesting and disrupting the cells, the proteins were separated by SDS-PAGE and biotinylated BCCP was detected by fluorography (Marini et al., 1995).

Fatty acid synthesis in vivo. $B$. subtilis cultures were grown to $\mathrm{OD}_{600} 0.5$ in $\mathrm{DS}$ medium and then labelled with $2.5 \mu \mathrm{Ci}$ $(92.5 \mathrm{kBq})$ of either $\left[{ }^{14} \mathrm{C}\right]$ acetate $\quad\left(56.7 \mathrm{mCi} \mathrm{mmol}^{-1}\right.$; $\left.2 \cdot 1 \mathrm{GBq} \mathrm{mmol}^{-1}\right)$ or $\left[{ }^{14} \mathrm{C}\right]$ isoleucine $\left(342 \mathrm{mCi} \mathrm{mmol}^{-1}\right.$; $12.7 \mathrm{GBq} \mathrm{mmol}^{-1}$ ) for $1 \mathrm{~h}$ as previously described (Grau \& de Mendoza, 1993; Grau et al., 1994). Cellular lipids were extracted from whole cells, saponified, and the amount of $\left[{ }^{14} \mathrm{C}\right]$ acetate incorporated into long-chain fatty acids was determined as previously described (Grau \& de Mendoza, 1993; Grau et al., 1994; de Mendoza et al., 1983).

Fatty acid supplementation studies and fatty acid analysis. Fatty acid supplementation studies and fatty acid analysis were performed with cells grown in the mineral salts medium of Spizizen (1958) supplemented with glucose $(0.5 \%)$, casein hydrolysate $(0.1 \%)$ and tryptophan $\left(40 \mu \mathrm{g} \mathrm{m}^{-1}\right)$. For fatty acid supplementation studies the growth media contained $0.075 \%$ Brij 58. The different fatty acids were added at a final concentration of $16 \mu \mathrm{g} \mathrm{ml}^{-1}$. For fatty acid analysis cell pellets were lyophilized, resuspended in $1 \mathrm{ml} 5 \%(\mathrm{v} / \mathrm{v}) \mathrm{HCl}$ in methanol and heated for $2.5 \mathrm{~h}$ at $85^{\circ} \mathrm{C}$; the resulting fatty acid methyl esters were extracted as described elsewhere (Grau \& de Mendoza, 1993; Grau et al., 1994). Fatty acid methyl esters were analysed by gas chromatography using a Konik KNK3000 gas chromatograph. Fatty acid methyl esters were separated isothermally at $180^{\circ} \mathrm{C}$ using an HP-FFAP column (25 m $\times 0.2 \mathrm{~mm}$ internal diameter; Hewlett Packard). The injection port and detector temperature were operated at 250 and $300^{\circ} \mathrm{C}$ respectively. Fatty acid methyl esters were identified by comparing their retention times with those of standards of authentic branched-chain and straight-chain fatty acids (Sigma). Total fatty acids were quantified by comparison with a known amount of decanoic acid added prior to methanolysis.

Spore formation and germination. The GMP synthetase inhibitor decoyinine was added to a final concentration of $1.0 \mathrm{mg} \mathrm{m}{ }^{+}$to initiate sporulation in S7 medium (Nicholson \& Setlow, 1990). Spore titres were determined by heating dilutions of the culture to $80^{\circ} \mathrm{C}$ for $20 \mathrm{~min}$ and plating on $\mathrm{LB}$ medium supplemented with $1 \mathrm{mM}$ IPTG. For assays of spore germination, B. subtilis strains JH642 and DL2 were induced to sporulate in DS medium and the resultant spores washed as described by Nicholson \& Setlow (1990). Immediately prior to germination, spore suspensions were heat-activated at $70^{\circ} \mathrm{C}$ for $60 \mathrm{~min}$ and cooled on ice (Nicholson \& Setlow, 1990; Venkatasubramanian \& Johnstone, 1993). Germination of spores was induced in two systems as follows: (i) the ALA system, in which the spores were germinated for $80 \mathrm{~min}$ at $37^{\circ} \mathrm{C}$ in $50 \mathrm{mM}$ Tris $/ \mathrm{HCl}, \mathrm{pH} 8.0$, by the addition of $1 \mathrm{mM}$ (final concentration) L-alanine; (ii) the GFAK system, in which the spores were germinated for $80 \mathrm{~min}$ at $37^{\circ} \mathrm{C}$ in $50 \mathrm{mM}$ Tris/ $\mathrm{HCl}, \mathrm{pH} 8 \cdot 0$, by the addition of $5.6 \mathrm{mM}$ glucose, $5.6 \mathrm{mM}$ fructose, $30 \mathrm{mM}$ L-asparagine and $67 \mathrm{mM} \mathrm{KCl}$. The extent of germination in spore suspensions was determined as the percentage of the control loss of $\mathrm{OD}_{600}$ and the rate of germination was measured as the maximum rate of loss of $\mathrm{OD}_{600} \mathrm{~min}^{-1}$ divided by the initial $\mathrm{OD}_{600}$, as described by 
Venkatasubramanian \& Johnstone (1993). Outgrowth studies were carried out in enriched media supplemented with glucose and L-alanine (Venkatasubramanian \& Johnstone, 1993).

\section{RESULTS}

\section{Construction of a strain with the accBC operon under control of the spac promoter}

We have recently reported that the $a c c B$ and $a c c C$ genes encoding the $\mathrm{BCCP}$ and $\mathrm{BC}$ subunits of $\mathrm{ACC}$ form part of a single-copy operon located $220^{\circ}$ downstream of spolllA on the B. subtilis chromosome (Marini et al., 1995). Many attempts to interrupt either the $a c c B$ or the acc $\mathrm{C}$ gene by a simple crossover recombination event using integrative plasmids containing internal fragments of each gene were unsuccessful. Our failure to interrupt accBC in the chromosome of $B$. subtilis, combined with the fact that ACC catalyses the first committed step in fatty acid (and hence lipid) synthesis, suggests an essential role for these gene products. In order to test this possibility, the single chromosomal copy of the $a c c B C$ operon (Marini et al., 1995) was placed under spac promoter control. To do this, a fragment containing the $5^{\prime}$ end of the $a c c B$ gene (Fig. 1) was cloned along with the spac promoter and the lacl gene into an integrational plasmid vector to give pDL1. Transformation of $B$. subtilis JH642 with this plasmid only yielded transformants in the presence of IPTG. One of these transformants was designated DL2. The integration of plasmid pDL1 by Campbell insertion into DL2 placed the single copy of $a c c B C$ downstream from the spac promoter (Fig. 1). The integration of pDL1 by this event was confirmed by Southern blot hybridization experiments. As shown in Fig. 2, in an EcoRI digest of wildtype DNA, $a c c B$ and part of $a c c C$ are located in a single $1.32 \mathrm{kbp}$ fragment. In strain DL2, the fragment was split into a $6.5 \mathrm{~kb}$ fragment carrying the truncated gene of $a c c B$ and a $1.3 \mathrm{~kb}$ fragment that was joined to the $s p a c$ promoter (Fig. 2).

\section{Growth characteristics of DL2 and effects of BCCP depletion on lipid synthesis}

As shown in Fig. 3, normal growth of strain DL2 depended on the presence of IPTG. In the absence of IPTG, cell growth was strongly affected. Growth returned to the normal level upon addition of $1 \mathrm{mM}$ IPTG, which derepresses the expression of $a c c B$. However, when cultures were inoculated at initial $\mathrm{OD}_{600} 0 \cdot 1-0 \cdot 2$, growth of strain DL2 reached stationary phase in DS medium without IPTG after about $24 \mathrm{~h}$ at $37^{\circ} \mathrm{C}$. This growth was probably due to leakiness of the control system (Beall \& Lutkenhaus, 1991; Micka et al., 1991) since strain DL2 transformed with pHlacl, a high-copynumber plasmid containing the lacl gene, was unable to grow in the absence of IPTG (Fig. 3). The addition of $1 \mathrm{mM}$ IPTG restablished the growth of this strain to normal levels (data not shown).

To determine the effect of IPTG on the BCCP content, we exploited the fact that this $17 \cdot 2 \mathrm{kDa}$ biotinylated subunit of ACC can be easily detected in crude cell extracts previously labelled with $\left[{ }^{3} \mathrm{H}\right]$ biotin (Marini et al., 1995). To this end, strain DL2 was labelled with biotin in medium supplemented or unsupplemented with IPTG and crude cell extracts were examined by SDS-PAGE and autoradiography. The levels of BCCP of strain DL2 growing exponentially in the presence of $1 \mathrm{mM}$ IPTG (Fig. 4a, lane 1) were comparable with the wild-type levels (Fig. 4a, lane 3). However, in the absence of the inducer the BCCP content of strain DL2 decreased to about $15 \%$ of the levels observed in IPTGsupplemented cells (Fig. 4a, lane 2; Fig. 4b). In addition to BCCP, B. subtilis contains an $85 \mathrm{kDa}$ biotinylated protein (Marini et al., 1995). The biotinylation level of this protein was comparable in strain DL2 growing in media with or without IPTG (Fig. 4a, lanes 1-3), showing that the lack of IPTG specifically affects the synthesis of BCCP rather than the transport of biotin or the activity of the biotin apoprotein ligase.

To determine the effect of BCCP depletion on total fatty acid synthesis, we analysed the synthesis of radioactive fatty acids in cultures of strain DL2 growing exponentially in the presence of $\left[{ }^{14} \mathrm{C}\right]$ acetate in media supplemented or unsupplemented with $1 \mathrm{mM}$ IPTG. In the absence of IPTG we observed about $90 \%$ inhibition of the incorporation of $\left[{ }^{14} \mathrm{C}\right]$ acetate into the phospholipids of strain DL2 (8628 c.p.m. in the presence of IPTG, 489 c.p.m. in its absence). Similar results were obtained when cells were labelled with $\left[{ }^{14} \mathrm{C}\right]$ isoleucine (21372 c.p.m. in the presence of IPTG, 853 c.p.m. in its absence); isoleucine is the precursor of 12-methyltetradecanoic acid (anteiso-15), one of the major branched-chain fatty acids of $B$. subtilis (de Mendoza et al., 1993). All these results suggest an essential role of the $a c c B C$ operon gene products for growth in $B$. subtilis. They also show that the fully induced spac promoter could provide sufficient BCCP and BC for fatty acid synthesis and normal growth.

\section{The growth defect of DL2 is corrected in the presence of long branched-chain fatty acids}

The above results indicated that the mutant strain DL2 has a conditional decreased synthesis of the BCCP and BC subunits of ACC. Since ACC catalyses the synthesis of malonyl-CoA, the primer for the elongation step of fatty acids by the condensing enzyme(s) (de Mendoza $e t$ al., 1993; Magnuson et al., 1993; Rock \& Cronan, 1996), we investigated whether this defect could be corrected by growing DL2 in the presence of long branched-chain fatty acids. Strain DL2 grew poorly in an IPTG-free medium, reaching only $\mathrm{OD}_{600} 0.5$ after $7 \mathrm{~h}$ growth (Fig. 3). However, when the medium was supplemented with different long branched-chain fatty acids, growth was restored to the level of IPTGsupplemented cultures (Fig. 3). The growth of the mutant was slightly stimulated by the straight-chain fatty acids decanoic acid or palmitic acid, but the addition of palmitoleic acid, a straight-chain unsaturated fatty acid resulted in cell lysis (Fig. 3). 

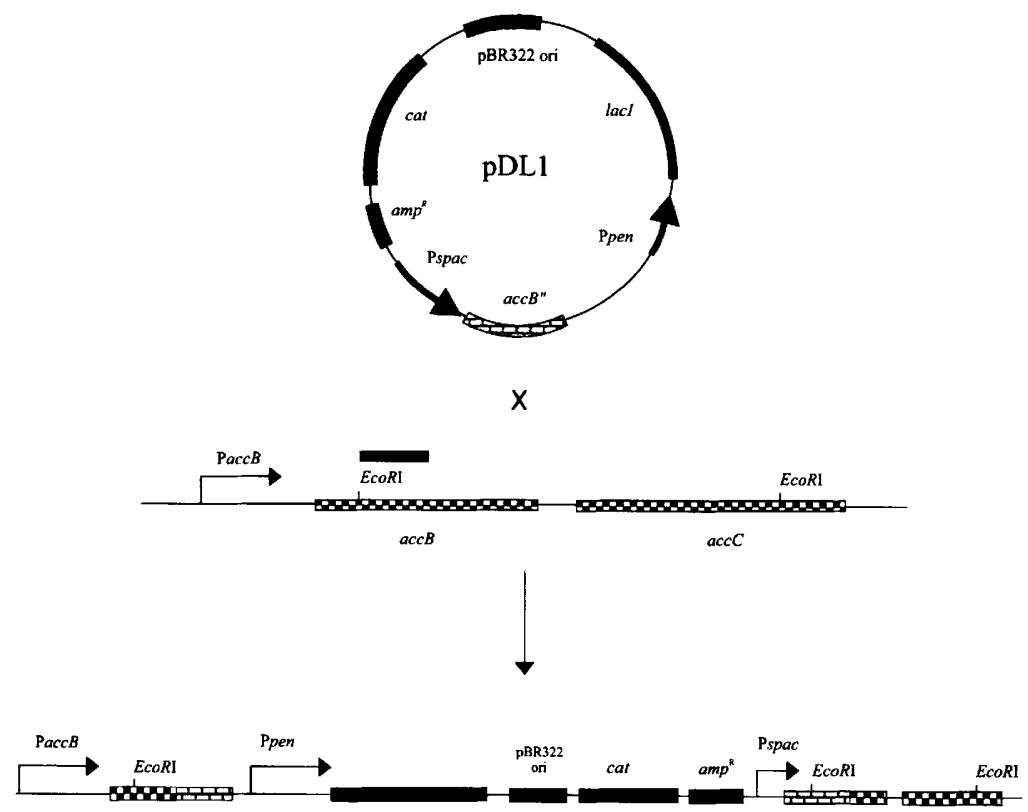

$a^{a c B} B^{\prime \prime}$

Lacl

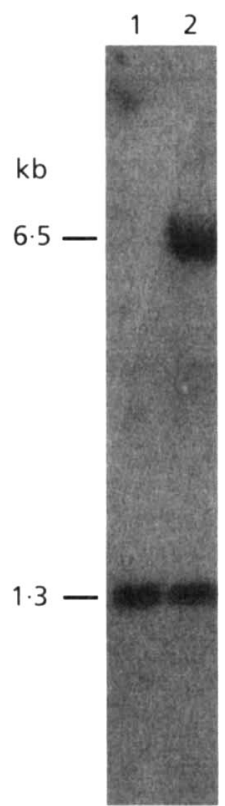

Fig. 2. Characterization of $B$ subtilis $D L 2$ by Southern hybridization. Chromosomal DNA of $B$. subtilis wild-type (lane 1) and DL2 (lane 2) was digested with EcoRI. The cleaved product was separated on a $0.8 \%$ agarose gel, transferred to nitrocellulose and hybridized with a probe specific for acCB (Fig. 1). The positions of the accBC genes in the wild-type $(1.3 \mathrm{~kb})$ and in strain $D L 2$, containing the truncated copy $(6.5 \mathrm{~kb})$ and the spac accBC fusion $(1.3 \mathrm{~kb})$, are indicated.

\section{Fatty acid content of DL2}

Table 1 shows the fatty acid composition of strain DL2 grown without supplements or in the presence of either IPTG or 13-methyltetradecanoic acid (iso-15:0). The most abundant fatty acid in strain DL2 grown with

accB $\quad$ acc $C$

Fig. 1. Construction of IPTG-dependent $B$. subtilis strain DL2, with the acCBC operon under spac promoter control. Plasmid pDL1, which contains the RBS and the first 124 codons of the $\operatorname{acc} B C$ gene downstream of the spac promoter, was used to transform wild-type strain $\mathrm{JH} 642$ to $\mathrm{Cm}^{\mathrm{R}}$ in the presence of $1.0 \mathrm{mM}$ IPTG to give strain DL2. Campbell insertion of this plasmid places the accBC operon under spac promoter control. The black bar above the chromosomal copy of $a c c B$ denotes the restriction fragment used to confirm the integration of $\mathrm{pDL} 1$ by Southern blot hybridization, as shown in Fig. 2. Only the position of the EcoRI sites in the $a c c B C$ operon is indicated. 


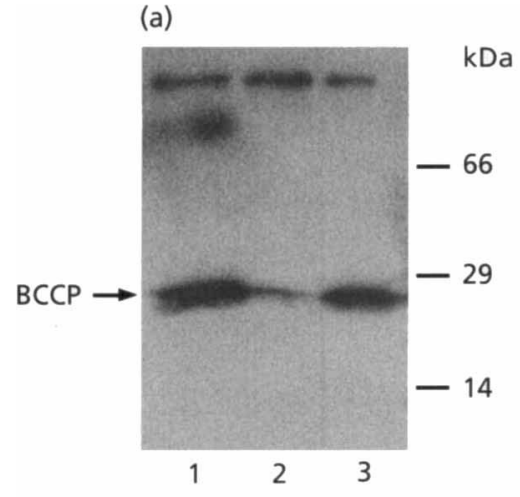

(b)

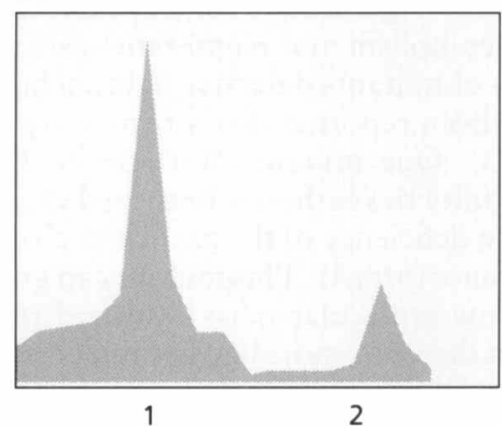

Fig. 4. Characterization of $B$. subtilis $\mathrm{DL} 2$ bearing the Pspac-accBC fusion. (a) Effect of IPTG on synthesis of biotinylated BCCP. Strain DL2 growing with IPTG (lane 1) or without IPTG (lane 2), or the wild-type strain (lane 3), was grown for several generations at $37^{\circ} \mathrm{C}$ in DS medium in the presence of $\left[{ }^{3} \mathrm{H}\right]$ biotin as described in Methods. The proteins were separated by SDS-PAGE $(15 \%, w / v$, acrylamide) and the bands were detected by fluorography. (b) Densitometric tracing of the BCCP radioactive bands pictured in lanes 1 and 2 , respectively, of (a).

mented with iso-15:0, the fraction of this fatty acid increased about sixfold (Table 1). This result indicates that iso-15:0, a fatty acid that restores normal growth of strain DL2 (Fig. 3), is incorporated into membrane phospholipids of the mutant strain. It should be noted that the total content of fatty acids of strain DL2 growing in an IPTG-free medium was about $30 \%$ of the total fatty acid content of cells grown in a medium supplemented with IPTG or with iso-15:0. In Table 1 it is shown that the ratio of iso to anteiso acyl chains is increased in strain DL2 (growing with or without IPTG) when compared to the wild-type strain JH642. The different fatty acid composition observed in DL2 could be due to the fact that in this strain the transcription of accBC is unregulated. In E. coli the transcription of the $a c c B C$ operon is tightly regulated, possibly by a positive activator (Li \& Cronan, 1993).

\section{Sporulation and germination of DL2}

We determined that fatty acid synthesis is required for spore formation when sporulation is induced with decoyinine in the early exponential phase of growth. Typically, the sporulation efficiency was between $50 \%$ and $70 \%$ (usually corresponding to approximately $3 \times 10^{7}$ spores $\mathrm{ml}^{-1}$ ) for the wild-type strain JH642 or for DL2 growing in the presence of IPTG. The sporulation frequency of strain DL2 was typically reduced 100-fold, with no effect on cell viability, when IPTG was omitted from the sporulation medium. These results show that under these assay conditions inhibition of fatty acid synthesis dramatically reduces spore formation.

Spores of B. subtilis can be induced to germinate by incubation in L-alanine (the ALA pathway) or in a combination of glucose, fructose, $\mathrm{L}$-asparagine and $\mathrm{K}^{+}$ (the GFAK pathway) (Foster \& Johnstone, 1989, 1990). As shown in Fig. 5, when germination was measured by monitoring the decrease in $\mathrm{OD}_{600}$ of a spore suspension upon the addition of $10 \mathrm{mM}$ L-alanine, spores of strain DL2 obtained without IPTG responded to the germinant. However, the $\mathrm{OD}_{600}$ values of the spore suspension decreased more slowly compared either with spores of DL2 obtained with IPTG or with the wild-type strain, as shown in Fig. 5. Similar results were obtained when germination was measured in the GFAK pathway

Table 1. Effect of growth supplement on the fatty acid content of B. subtilis strain DL2

Cells were grown in the media described in Methods at $37^{\circ} \mathrm{C}$ and harvested at $\mathrm{OD}_{600} 0.5$.

\begin{tabular}{|c|c|c|c|c|c|c|c|c|c|}
\hline \multirow[t]{2}{*}{ Strain } & \multirow[t]{2}{*}{ Supplement } & \multicolumn{6}{|c|}{ Percentage $(w / w)$ of fatty acid type } & \multirow{2}{*}{$\begin{array}{l}\text { Relative amount of } \\
\text { fatty acids }(\%)^{+}\end{array}$} & \multirow{2}{*}{$\begin{array}{c}\text { Ratio } \\
\text { iso: anteiso }\end{array}$} \\
\hline & & iso- 15 & anteiso-15 & iso-16 & $n-16$ & iso- 17 & anteiso- 17 & & \\
\hline DL2 & IPTG & 38 & 18 & 15 & 14 & 2 & 10 & 112 & 1.96 \\
\hline DL2 & - & 4 & 24 & 33 & 14 & 14 & 9 & 39 & $1 \cdot 54$ \\
\hline DL2 & Iso-15 & 25 & 17 & 27 & 14 & 2 & 11 & 118 & $1 \cdot 92$ \\
\hline JH642 & - & 18 & 40 & 14 & 14 & 11 & 2 & 100 & $1 \cdot 0$ \\
\hline
\end{tabular}

"Data are the means of at least three experiments with the mean error in percentage values of fatty acids being less than $5 \%$. The values are expressed as percentage of total fatty acid methyl esters analysed.

†Total cellular fatty acid was measured as described in Methods, using decanoic acid as an internal standard. Amounts are related to that in wild-type strain $\mathrm{JH} 642$ (taken as $100 \%$ ). 


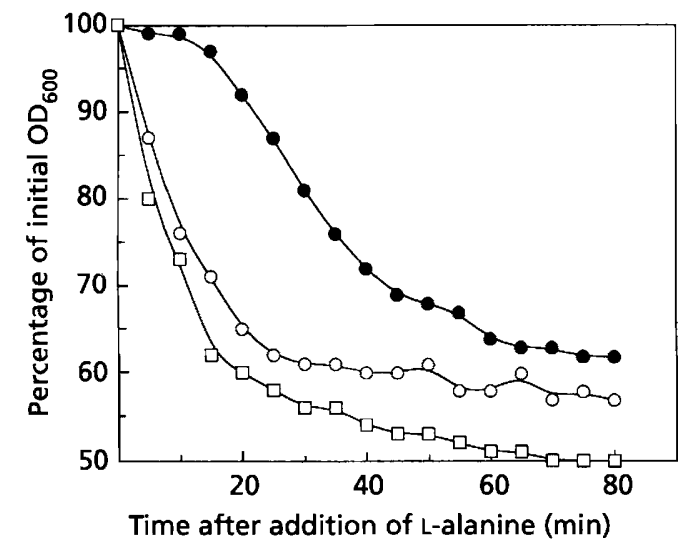

Fig. 5. Germination of spores of DL2 obtained in the presence or in the absence of IPTG in the ALA system. JH642 spores ( $\square$ ) or DL2 spores obtained with IPTG (O) or without IPTG (O) were treated with L-alanine and the percentage of initial $O D_{600}$ determined as described in Methods.

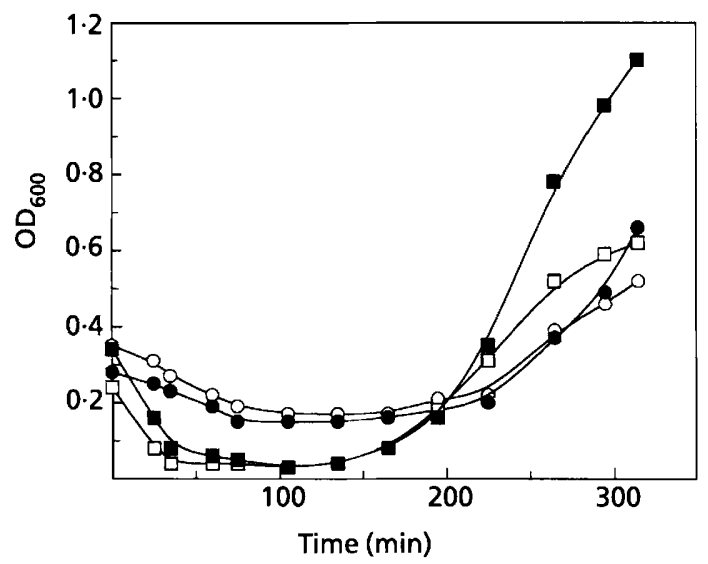

Fig. 6. Outgrowth of spores of DL2 obtained in the presence or in the absence of IPTG. $\square, \square$, Spores obtained with 1 mM IPTG; 0 , spores obtained without IPTG. Spores were germinated either in medium supplemented with 1 mM IPTG $(\square, 0)$ or in medium devoid of IPTG $(\square, O)$.

(data not shown). Lineweaver-Burk plots of L-alanine concentration against germination rate (Venkatasubramanian \& Johnstone, 1993) indicated that spores of strain DL2 obtained without IPTG have a $K_{\mathrm{m}}$ of $91 \mu \mathrm{M}$ for the germinant, while the $K_{\mathrm{m}}$ of spores obtained with IPTG was $56 \mu \mathrm{M}$ (data not shown). This suggests that spores produced during conditions of limited lipid synthesis possess a receptor responding to L-alanine in the ALA pathway with a decreased affinity for the ligand that triggers spore germination.

Outgrowth studies were carried out in medium supplemented with glucose and L-alanine. In this medium, spores of strain DL2 obtained in media supplemented with IPTG showed the expected drop in $\mathrm{OD}_{600}$ for the first hour of incubation (Fig. 6). After about $2 \mathrm{~h}$ at $37^{\circ} \mathrm{C}$, the $\mathrm{OD}_{600}$ of DL2 cultures began to rise; further growth was dependent on the presence of IPTG (Fig. 6). On the other hand, the rise in $\mathrm{OD}_{600}$ that followed the germination of spores obtained in an IPTG-free medium was significantly delayed when compared with spores formed in the presence of the inducer, irrespective of whether the growth medium was supplemented with IPTG (Fig. 6).

\section{DISCUSSION}

Although $B$. subtilis is genetically the best-characterized Gram-positive organism, it has not been fully exploited for lipid metabolism and membrane research, and only two classes of mutants defective in branched-chain fatty acids have been reported (for a review see de Mendoza et al., 1993). One mutant (Willecke \& Pardee, 1971) lacks the ability to synthesize branched-chain fatty acids because of a deficiency of the branched-chain 2-ketoacid dehydrogenase $(b f m A)$. This mutant can grow on a wide variety of low-molecular-mass branched and cyclic fatty acids, but it does not sporulate because it is also deficient in pyruvate dehydrogenase activity (Willecke $\&$ Pardee, 1971). To investigate the effects of membrane changes on growth and sporulation in a strain having normal pyruvate dehydrogenase activity, Boudreaux et al. (1981) isolated a $b f m B$ mutant that results in an acylCoA:ACP transacylase with low affinity for branched acyl-CoA precursors. The consequence of this mutation is a growth requirement for a branched-chain fatty acid precursor (Boudreaux et al., 1981). However, in our hands the $b f m B$ mutant did not show the reported phenotype and precluded any manipulation to study lipid synthesis during growth and sporulation. Thus, studies aimed at identifying specific functions of branched-chain fatty acids have been difficult because of a lack of experimental models that allow the manipulation of fatty acids during the cell cycle. With the aim of studying the role of fatty acid synthesis in growth and differentiation in B. subtilis, we constructed a strain bearing a copy of the $a c c B C$ operon under the control of the spac promoter. Cells containing the Pspac-accBC fusion had reduced BCCP levels and grew much more slowly than the same cells in the presence of $1 \mathrm{mM}$ IPTG. The reduction in the levels of BCCP drastically reduced de novo fatty acid synthesis and the total content of cellular fatty acids (Table 1). The growth of DL2 was restored by supplying exogenous long-chain fatty acids in the medium, indicating that the inducerdependent phenotype was specifically related to a conditional block in fatty acid biosynthesis. In agreement with the fact that iso and anteiso branched-chain fatty acids are the dominant acyl components of the lipids of B. subtilis (de Mendoza et al., 1993), the growth of DL2 was specifically stimulated by these fatty acids (Fig. 3). While the growth of the mutant was slightly stimulated by decanoic or palmitic acid, which are straight-chain saturated fatty acids, the addition of palmitoleic acid, a straight-chain unsaturated fatty acid, 
resulted in cell lysis (Fig. 3). These results suggest that straight-chain fatty acids are not incorporated efficiently into $B$. subtilis membrane phospholipids or that branched-chain fatty acids may play some specific role that can not be replaced by straight-chain fatty acids.

There are several lines of evidence indicating that iso and anteiso acyl chains play the biological role of straight saturated and unsaturated acyl chains, respectively, in a membrane containing large amounts of these compounds (de Mendoza et al., 1993). However, although the ratio of iso to anteiso acyl chains in DL2 (growing with IPTG) is significantly higher than the wild-type strain JH642, these strains do not show a difference in growth rate. Thus it is evident that $B$. subtilis can grow normally despite the fact that more than $50 \%$ of the fatty acids of the membrane lipids are iso fatty acids.

DL2 cells growing without IPTG showed a marked decrease in iso-15 fatty acid content, with a concomitant increase in iso-16 and iso-17 fatty acids, while the percentage of anteiso fatty acids remained almost unchanged. Since the ratio of iso to anteiso acyl chains only decreases about $25 \%$ in the absence of IPTG (Table 1) it seems that the major consequence of reducing the ACC levels is to decrease the total amount of membrane fatty acids rather than to change the physical state of the membrane phospholipid acyl chains. It is worth noting that although most cells maintain a constant ratio of proteins (both whole cell and membrane), the synthesis of membrane proteins is not coupled to phospholipid synthesis (for a review see Cronan \& Rock, 1987). For example, by using E. coli mutants blocked in the first step of phospholipid synthesis, McIntyre \& Bell (1975) found that both the inner membrane and the outer membrane became enriched with protein. This work demonstrates that the membrane is not normally saturated with protein and that the synthesis of membrane phospholipids is not required for the synthesis and insertion of bulk membrane proteins. In addition the protein-enriched membranes were not lethal to the cell, and the enrichment was quickly normalized upon the restoration of phospholipid synthesis (McIntyre et al., 1977). Taking into account these considerations, $B$. subtilis cells growing with a limited supply of fatty acids (and hence phospholipids) should have membranes with an increased ratio of protein to phospholipids.

IPTG starvation with the Pspac-accBC fusion strongly suggested that the $\operatorname{acc} B C$ operon was essential during exponential growth, although the mutant could reach stationary phase after about $24 \mathrm{~h}$ incubation at $37^{\circ} \mathrm{C}$. The reduced growth in the absence of IPTG is probably due to leakiness of the inducible spac promoter since supplying the LacI repressor in trans totally abolished the growth of strain DL2 in the absence of the inducer (Fig. 3).

The major changes associated with membrane differentiation during spore formation in B. subtilis are (i) the formation of an asymmetric sporulation septum that gives rise to a sporangium containing unequal progeny called the prespore and the mother cell and (ii) the process of engulfment in which these two cell types collaborate in the conversion of the postseptation sporangium to a cell-within-a-cell structure (for recent reviews see Errington, 1993; Stragier \& Losick, 1996). Although little is understood about the molecular mechanism of these morphological changes (Stragier $\&$ Losick, 1996) it is assumed that these differentiation processes require abundant de novo lipid synthesis and membrane components (Feucht et al., 1996; Piggot et al., 1994; Stragier \& Losick, 1996). To determine the effect of a deficiency in fatty acid supply on sporulation we used the drug decoyinine, which induces spore formation by inhibiting GTP biosynthesis (Mitani et al., 1977). In a defined medium with decoyinine as inducer of sporulation, the mutant strain sporulated poorly without IPTG. These results are consistent with a requirement of active lipid synthesis for spore formation. Clearly, it remains to be determined which stage of sporulation is blocked when there is a reduced rate of fatty acid synthesis.

Transformation of a dormant resistant bacterial endospore into a metabolically active vegetative cell involves a complex series of interrelated biochemical events (for reviews see Foster \& Johnstone, 1989, 1990; Moir \& Smith, 1990). This germination response is triggered in spores by germinants. However, the mechanism by which the germinants interact with spore components to initiate germination is not known (Foster \& Johnstone, 1989, 1990; Moir \& Smith, 1990). It has been proposed that alanine acts as a germinant by penetrating the spore as far as the outer surface of the inner membrane, where it interacts with a receptor complex; this interaction results in the transduction of the germination stimulus to initiate a cascade of subsequent hydrolytic reactions (Moir \& Smith, 1990). Several lines of evidence indicate that the putative receptor is in a hydrophobic environment and it has been suggested that spore germination could be dependent on membrane lipid composition (Foster \& Johnstone, 1989). We show here that spores formed by the mutant strain DL2 in the presence of IPTG germinated just as well as the wildtype in the ALA and the GFAK systems. However, the germination of spores obtained in a medium devoid of IPTG was significantly delayed in these two germination systems, supporting the proposal that part of the commitment process appears to be membrane-associated (Foster \& Johnstone, 1989; Nicholson \& Setlow, 1990). In addition, we found that the L-alanine receptor has decreased affinity for its ligand under conditions of limited fatty acid synthesis. However, this result does not explain the slower germination rate of spores obtained under conditions of lipid starvation, since spore germination was assayed at saturating germinant concentrations. Thus, a shortage of fatty acids should affect other components of the cascade events comprising the germination response (Foster \& Johnstone, 1990).

We also show here that spores formed without IPTG initiated outgrowth much more slowly than spores 
formed in the presence of IPTG, irrespective of the presence of IPTG in the spore germination medium (Fig. 6). These results suggest that membrane lipids could play an important role in the initiation of outgrowth of germinating spores.

The decreased synthesis of fatty acids might modify the chemical nature of the membrane, thereby producing the observed phenotypes in the B. subtilis cell cycle. Alternatively, the phenotypes may be an indirect and possibly pleiotropic consequence of a sick cell that fails to assemble important membrane components necessary for differentiation. Therefore, ACC activity seems to be an essential component of spore formation and is also required for the germination process, as well as to initiate outgrowth of germinating spores.

\section{ACKNOWLEDGEMENTS}

Decoyinine was a gift from Upjohn Company. We thank D. Henner and T. Msadek for providing plasmids pDH88 and $\mathrm{pH}$ laci respectively. We thank $\mathrm{C}$. Rúbeda for her cooperation with fatty acid analysis, $M$. Arévalo for outstanding technical assistance, and M. Perego for help in the design of synthetic oligonucleotides. Gratitude is also expressed to J. A. Hoch (The Scripps Research Institute, La Jolla, CA, USA) who kindly provided facilities for the construction of plasmid pDL1 during a visit of D. de M. as a fellow of the John Simon Guggenheim Memorial Foundation (USA). This work was supported by the European Communities grant 937004 AR, Fundación Antorchas (Argentina) and Consejo Nacional de Investigaciones Científicas y Técnicas de Argentina (CONICET). C.P. is a fellow from CONICET. P.M. and D. de M. are Career Investigators from Consejo de Investigaciones de la Universidad Nacional de Rosario and CONICET, respectively.

\section{REFERENCES}

Arantes, O. \& Lereclus, D. (1991). Construction of cloning vectors for Bacillus thuringiensis. Gene 108, 115-119.

Beall, B. \& Lutkenhaus, J. (1991). FtsZ in Bacillus subtilis is required for vegetative septation and for asymmetric septation during sporulation. Genes Dev 5, 447-455.

Boudreaux, D. P., Eisenstat, E., Ijima, T. \& Freese, E. (1981). Biochemical and genetic characterisation of an auxotroph of Bacillus subtilis altered in acyl-CoA acyl-acyl-carrier-proteintransacylase. Eur J Biochem 12, 496-501.

Cronan, J. E., Jr \& Rock, C. O. (1987). Biosynthesis of membrane lipids. In Escherichia coli and Salmonella typhimurium: Cellular and Molecular Biology, pp. 474-497. Edited by F. C. Neidhart, J. L. Ingrahan, K. B. Low, B. Magasanik, M. Schaechter \& H. E. Umbarger. Washington, DC: American Society for Microbiology. Errington, J. (1993). Sporulation in Bacillus subtilis: regulation of gene expression and control of morphogenesis. Microbiol Rev 57, 1-33.

Feucht, A., Magnin, T., Yudkin, M. D. \& Errington, J. (1996). Bifunctional protein required for asymmetric cell division and cell-specific transcription in B. subtilis. Genes Dev 10, 794-803.

Foster, S. J. \& Johnstone, K. (1989). The trigger mechanism of bacterial spore germination. In Regulation of Prokaryotic Development, pp. 89-109. Edited by I. Smith, R. A. Slepecky \& P. Setlow. Washington, DC: American Society for Microbiology.
Foster, S. J. \& Johnstone, K. (1990). Pulling the trigger: the mechanism of bacterial spore germination. Mol Microbiol 4, 137-141.

Gardiol, D., Gramajo, H., Hirschbein, L. \& de Mendoza, D. (1993). Application of the mini-Mu phage for the isolation of lac transcriptional fusions in Bacillus subtilis genes. Gene 123, 39-44.

Grau, R. \& de Mendoza, D. (1993). Regulation of the synthesis of unsaturated fatty acids by growth temperature in Bacillus subtilis. Mol Microbiol 8, 535-542.

Grau, R., Gardiol, D., Glikin, G. \& de Mendoza, D. (1994). DNA supercoiling and thermal regulation of unsaturated fatty acid synthesis in Bacillus subtilis. Mol Microbiol 11, 933-941.

Henner, D. J. (1990). Inducible expression of regulatory genes in Bacillus subtilis. Methods Enzymol 185, 223-228.

Kim, K. H. (1983). Regulation of acetyl-CoA carboxylase. Curr Top Cell Regul 22, 143-172.

Li, S. J. \& Cronan, J. E., Jr (1992a). The gene encoding the biotin carboxylase subunit of acetyl CoA carboxylase. J Biol Chem 267 , 855-863.

Li, S. J. \& Cronan, J. E., Jr (1992b). The genes encoding the two carboxyltransferase subunits of Escherichia coli acetyl CoA carboxylase. J Biol Chem 267, 16841-16487.

Li, S. J. \& Cronan, J. E., Jr (1993). Growth rate regulation of Escherichia coli acetyl coenzyme A carboxylase, which catalyses the first committed step in lipid biosynthesis. J Bacteriol 175, 332-340.

López-Casilla, F., Pape, M. E., Bai, D. H., Kuhn, N., Dixon, J. E. \& Kim, K. H. (1987). Preparation of functional acetyl-CoA carboxylase mRNA from rat mammary gland. Arch Biochem Biophys 257, 63-68.

Luo, X. \& Kim, K. H. (1990). An enhancer element in the housekeeping promoter for acetyl-CoA carboxylase gene. Nucleic Acids Res. 18, 3249-3254.

McIntyre, T. M. \& Bell, R. M. (1975). Mutants of Escherichia coli defective in membrane phospholipid synthesis. Effect of cessation of net phospholipid synthesis on cytoplasmic and outer membranes. J Biol Chem 250, 9053-9059.

McIntyre, T. M., Chamberlain, B. K., Webster, R. E. \& Bell, R. M. (1977). Mutants of Escherichia coli defective in membrane phospholipid synthesis. Effects of cessation and reinitiation of phospholipid synthesis on macromolecular synthesis and phospholipid turnover. J Biol Chem 252, 4487-4493.

Magnuson, K., Jackowski, S., Rock, C. O. \& Cronan, J. E., Jr (1993). Regulation of fatty acid biosynthesis in Escherichia coli. Microbiol Rev 57, 522-542.

Marini, P., Li, S. J., Gardiol, D., Cronan, J. E., Jr \& de Mendoza, D. (1995). The genes encoding the biotin carboxyl carrier protein and biotin carboxylase subunits of Bacillus subtilis acetyl coenzyme A carboxylase, the first enzyme of fatty acid synthesis. J Bacteriol 177, 7003-7006.

de Mendoza, D., Klages-Ulrich, A. \& Cronan, J. E., Jr (1983). Thermal regulation of membrane fluidity in Escherichia coli. Effects of overproduction of $\beta$-keto-acyl-acyl-carrier protein synthase I. J Biol Chem 258, 2098-2101.

de Mendoza, D., Grau, R. \& Cronan, J. E., Jr (1993). Biosynthesis and function of membrane lipids. In Bacillus subtilis and other Gram Positive Bacteria: Physiology, Biochemistry and Molecular Biology, pp. 411-421. Edited by R. Losick, A. L. Sonenshein \& J. A. Hoch. Washington, DC: American Society for Microbiology.

Micka, B., Groch, N., Heinemann, U. \& Marahiel, M. A. (1991).

Molecular cloning, nucleotide sequence, and characterisation of 
the Bacillus subtilis gene encoding the DNA-binding protein Hbsu. J Bacteriol 173, 3191-3198.

Mitani, T., Heinze, J. E. \& Freese, E. (1977). Induction of sporulation in Bacillus subtilis by decoyinine or hadamicin. Biochem Biophys Res Commun 115, 193-205.

Moir, A. \& Smith, D. A. (1990). The genetics of bacterial germination. Annu Rev Microbiol 44, 531-553.

Nicholson, W. L. \& Setlow, P. (1990). Sporulation, germination and outgrowth. In Molecular Biological Methods for Bacillus, pp. 391-429. Edited by C. R. Harwood \& S. M. Cutting. New York: Wiley.

Nikawa, J., Tanabe, T., Ogiwara, H., Shuba, T. \& Numa, S. (1979). Inhibitory effects of long-chain acyl-CoA analogues on rat liver acetyl-CoA carboxylase. FEBS Lett 102, 223-278.

Piggot, P. J., Bylund, J. E. \& Higgins, M. L. (1994). Morphogenesis and gene expression during sporulation. In Regulation of Bacterial Differentiation, pp. 113-137. Edited by P. J. Piggot, C. P. Moran \& P. Youngman. Washington, DC: American Society for Microbiology.

Rock, C. O. \& Cronan, J. E., Jr (1996). Escherichia coli as a model for the regulation of dissociable (type II) fatty acid biosynthesis. Biochim Biophys Acta 1302, 1-16.

Sambrook, J., Fritsch, E. F. \& Maniatis, T. (1989). Molecular
Cloning: a Laboratory Manual, 2nd edn. Cold Spring Harbor, NY: Cold Spring Harbor Laboratory.

Schaeffer, P., Millet, J. \& Aubert, J. (1965). Catabolite repression of bacterial sporulation. Proc Natl Acad Sci USA 54, 704-711.

Spizizen, J. (1958). Transformation of biochemically deficient strains of Bacillus subtilis by deoxyribonucleate. Proc Natl Acad Sci USA 44, 1072-1078.

Stragier, P. \& Losick, R. (1996). Molecular genetics of sporulation in Bacillus subtilis. Annu Rev Genet 30, 297-341.

Venkatasubramanian, P. \& Johnstone, K. (1993). Biochemical analysis of germination mutants to characterise germinant receptors of Bacillus subtilis 1604 spores. J Gen Microbiol 139, 1921-1926.

Volpe, J. J. \& Vagelos, P. R. (1976). Saturated fatty acid synthesis and its regulation. Physiol Rev 567, 339-417.

Wakil, S. J., Stoops, J. K. \& Joshi, V. C. (1983). Fatty acid synthesis and its regulation. Annu Rev Biochem 52, 537-579.

Willecke, K. \& Pardee, A. B. (1971). Fatty acid requiring mutants of Bacillus subtilis defective in branched chain keto acid dehydrogenase. J Biol Chem 246, 5264-5272.

Received 7 July 1997; revised 23 October 1997; accepted 8 December 1997. 